\title{
Interactions between uniformly magnetized spheres
}

\author{
Boyd F. Edwards, D. M. Riffe, Jeong-Young Ji, and William A. Booth \\ Department of Physics, Utah State University, Logan, Utah 84322
}

(Received 31 May 2015; accepted 14 December 2016)

\begin{abstract}
We use simple symmetry arguments suitable for undergraduate students to demonstrate that the magnetic energy, forces, and torques between two uniformly magnetized spheres are identical to those between two point magnetic dipoles. These arguments exploit the equivalence of the field outside of a uniformly magnetized sphere with that of a point magnetic dipole, and pertain to spheres of arbitrary sizes, positions, and magnetizations. The point dipole/sphere equivalence for magnetic interactions may be useful in teaching and research, where dipolar approximations for uniformly magnetized spheres can now be considered to be exact. The work was originally motivated by interest in the interactions between collections of small neodymium magnetic spheres used as desk toys. @ 2017 American Association of Physics Teachers.

[http://dx.doi.org/10.1119/1.4973409]
\end{abstract}

\section{INTRODUCTION}

Consider two permanent magnets of arbitrary shape. If each magnet has a nonzero dipole moment, then the dipole moments of these magnets will dominate their interactions at separations that are large compared with their sizes, and each magnet may be treated as if it were a point magnetic dipole. ${ }^{1}$ Dipolar fields and forces are often used as the starting point for analytical and numerical approximations of the forces between permanent magnets of various shapes. ${ }^{2-7}$

A uniformly magnetized sphere produces a magnetic field that is identical to its dipole field not just at large distances but everywhere outside of the sphere. ${ }^{8,9}$ One is thus naturally led to ask whether the forces and torques between two uniformly magnetized spheres are identical to those between two point dipoles, independent of their separation. Here we show that this is indeed the case.

This result has practical applications. Dipolar fields and forces have been used to approximate the interactions among assemblies of spherical nanoparticles ${ }^{10}$ and magnetic microspheres. ${ }^{11}$ Our results show that these approximations are, in fact, exact. In addition, small rare-earth magnetic spheres are used both in and out of the classroom to teach principles of mathematics, physics, chemistry, biology, and engineering. ${ }^{12-16}$ Our results enable simple dipole interactions to be used to model the dynamical interactions between these magnets. ${ }^{17,18}$

Previous calculations of the force between two uniformly magnetized spheres have been carried out in three limiting geometries: (i) for magnetizations that are perpendicular to the line through the sphere centers, ${ }^{19}$ (ii) for parallel magnetizations that make an arbitrary angle with this line,${ }^{20}$ and (iii) for configurations with one magnetization parallel to this line and the other in an arbitrary direction. ${ }^{21}$ All three calculations yield forces that are identical to the force between two point magnetic dipoles. ${ }^{22-27}$ Unlike these calculations, however, our calculations rely on simple symmetry arguments and pertain to spheres of arbitrary sizes, positions, magnetizations, and magnetic orientations.

The force between two dipoles is noncentral. That is, this force is not generally directed along the line through the dipoles. While other noncentral magnetic forces violate Newton's third law, ${ }^{28-30}$ we show that the paired forces between magnetic dipoles obey this law. These forces therefore exert a net $\mathbf{r} \times \mathbf{F}$ torque on an isolated two-dipole system. As we show below, this torque is canceled by paired $\mathbf{m} \times \mathbf{B}$ torques, which are not equal and opposite. Thus, the angular momentum is conserved.

\section{POINT DIPOLE INTERACTIONS}

In this section, we review the interactions between point magnetic dipoles. The magnetic field at position $\mathbf{r}$ produced by a point dipole $\mathbf{m}$ located at the origin is given by ${ }^{31,32}$

$$
\mathbf{B}(\mathbf{m}, \mathbf{r})=\frac{\mu_{0}}{4 \pi}\left(\frac{3 \mathbf{m} \cdot \mathbf{r}}{r^{5}} \mathbf{r}-\frac{\mathbf{m}}{r^{3}}\right),
$$

for $r=|\mathbf{r}|$ satisfying $r>0$. This field can be obtained from the scalar potential

$$
\varphi(\mathbf{m}, \mathbf{r})=\frac{\mu_{0}}{4 \pi} \frac{\mathbf{m} \cdot \mathbf{r}}{r^{3}},
$$

via

$$
\mathbf{B}(\mathbf{m}, \mathbf{r})=-\nabla \varphi(\mathbf{r}) .
$$

Because $\boldsymbol{\nabla} \cdot \mathbf{B}=0$, the scalar potential $\varphi$ satisfies Laplace's equation

$$
\nabla^{2} \varphi=0 .
$$

We consider two dipoles, $\mathbf{m}_{1}$ and $\mathbf{m}_{2}$, that are, respectively, located at positions $\mathbf{r}_{1}$ and $\mathbf{r}_{2}$. From Eq. (1), the field produced by dipole $\mathbf{m}_{i}$ is

$$
\mathbf{B}_{i}(\mathbf{r})=\mathbf{B}\left(\mathbf{m}_{i}, \mathbf{r}-\mathbf{r}_{i}\right),
$$

where $i=1,2$, and where $\mathbf{r}-\mathbf{r}_{i}$ is the position vector relative to dipole $\mathbf{m}_{i}$ (see Fig. 1). Accordingly, the field of $\mathbf{m}_{i}$ evaluated at the location of $\mathbf{m}_{j}$ is

$$
\mathbf{B}_{i}\left(\mathbf{r}_{j}\right)=\frac{\mu_{0}}{4 \pi}\left(\frac{3 \mathbf{m}_{i} \cdot \mathbf{r}_{i j}}{r_{i j}^{5}} \mathbf{r}_{i j}-\frac{\mathbf{m}_{i}}{r_{i j}^{3}}\right),
$$

where $\mathbf{r}_{i j}=\mathbf{r}_{j}-\mathbf{r}_{i}$ is the position of $\mathbf{m}_{j}$ relative to $\mathbf{m}_{i}$, and $r_{i j}=\left|\mathbf{r}_{j}-\mathbf{r}_{i}\right|$.

The interaction energy between $\mathbf{m}_{j}$ and the magnetic field of $\mathbf{m}_{i}$ is given by 


$$
U_{i j}=-\mathbf{m}_{j} \cdot \mathbf{B}_{i}\left(\mathbf{r}_{j}\right)
$$

Inserting Eq. (6) into this expression gives

$$
U_{i j}=\frac{\mu_{0}}{4 \pi}\left[\frac{\mathbf{m}_{i} \cdot \mathbf{m}_{j}}{r_{i j}^{3}}-3 \frac{\left(\mathbf{m}_{i} \cdot \mathbf{r}_{i j}\right)\left(\mathbf{m}_{j} \cdot \mathbf{r}_{i j}\right)}{r_{i j}^{5}}\right] .
$$

Meanwhile, the force of $\mathbf{m}_{i}$ on $\mathbf{m}_{j}$ follows from

$$
\mathbf{F}_{i j}=-\nabla_{j} U_{i j}
$$

where $\nabla_{j}$ is the gradient with respect to $\mathbf{r}_{j}$. Making use of Eq. (8) then yields

$$
\begin{aligned}
\mathbf{F}_{i j}= & \frac{3 \mu_{0}}{4 \pi r_{i j}^{5}}\left[\left(\mathbf{m}_{i} \cdot \mathbf{r}_{i j}\right) \mathbf{m}_{j}+\left(\mathbf{m}_{j} \cdot \mathbf{r}_{i j}\right) \mathbf{m}_{i}\right. \\
& \left.+\left(\mathbf{m}_{i} \cdot \mathbf{m}_{j}\right) \mathbf{r}_{i j}-5 \frac{\left(\mathbf{m}_{i} \cdot \mathbf{r}_{i j}\right)\left(\mathbf{m}_{j} \cdot \mathbf{r}_{i j}\right)}{r_{i j}^{2}} \mathbf{r}_{i j}\right] .
\end{aligned}
$$

The first two terms in the square brackets are, respectively, parallel to $\mathbf{m}_{j}$ and $\mathbf{m}_{i}$. Consequently, $\mathbf{F}_{i j}$ is not central-it is not generally parallel to the vector $\mathbf{r}_{i j}$ between the dipoles.

Because of the symmetry between $i$ and $j$, Eqs. (8) and (10) imply that

$$
U_{21}=U_{12},
$$

and

$$
\mathbf{F}_{21}=-\mathbf{F}_{12}
$$

confirming that Newton's third law applies to the magnetic force between point magnetic dipoles, and ensuring that linear momentum is conserved in an isolated two-dipole system.

We now investigate the torque $\tau_{i j}$ of $\mathbf{m}_{i}$ on $\mathbf{m}_{j}$, which we write as having two contributions ${ }^{33}$

$$
\tau_{i j}=\tau_{i j}^{A}+\tau_{i j}^{B}
$$

The first arises from $\mathbf{m}_{j}$ residing in the field of $\mathbf{m}_{i}$, which is given by

$$
\tau_{i j}^{A}=\mathbf{m}_{j} \times \mathbf{B}_{i}\left(\mathbf{r}_{j}\right)
$$

The sum of paired torques (arising from the dipoles residing in the fields of the other)

$$
\begin{aligned}
\tau_{12}^{A}+\tau_{21}^{A}= & \frac{3 \mu_{0}}{4 \pi r_{12}^{5}}\left[\left(\mathbf{m}_{1} \cdot \mathbf{r}_{12}\right) \mathbf{m}_{2} \times \mathbf{r}_{12}\right. \\
& \left.+\left(\mathbf{m}_{2} \cdot \mathbf{r}_{12}\right) \mathbf{m}_{1} \times \mathbf{r}_{12}\right]
\end{aligned}
$$

is generally not equal to zero. Therefore, unlike the paired forces $\mathbf{F}_{12}$ and $\mathbf{F}_{21}$, the paired torques $\tau_{12}^{A}$ and $\tau_{21}^{A}$ are not generally equal and opposite. The second contribution to the torque arises from the force of $\mathbf{m}_{i}$ on $\mathbf{m}_{j}$

$$
\tau_{i j}^{B}=\mathbf{r}_{j} \times \mathbf{F}_{i j},
$$

and because $\mathbf{F}_{i j}$ is noncentral, the sum

$$
\tau_{12}^{B}+\tau_{21}^{B}=\mathbf{r}_{12} \times \mathbf{F}_{12}
$$

is also generally not equal to zero.

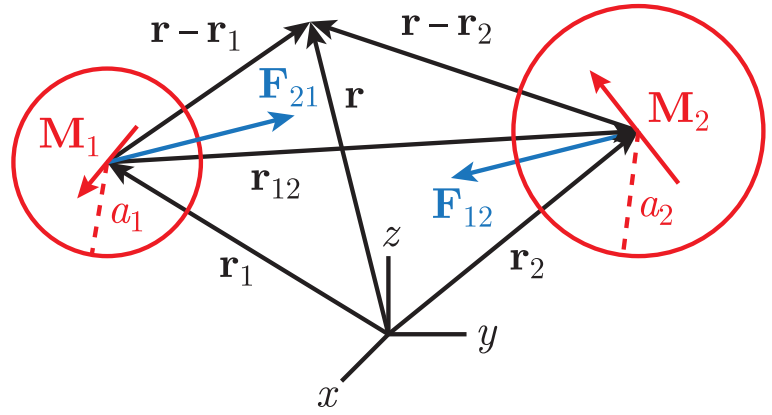

Fig. 1. Diagram showing two uniformly magnetized spheres with positions $\mathbf{r}_{1}$ and $\mathbf{r}_{2}$, radii $a_{1}$ and $a_{2}$, magnetizations $\mathbf{M}_{1}$ and $\mathbf{M}_{2}$, and paired noncentral magnetic forces $\mathbf{F}_{12}$ and $\mathbf{F}_{21}$. Also shown are an arbitrary position vector $\mathbf{r}$ and the relative position vectors $\mathbf{r}-\mathbf{r}_{1}, \mathbf{r}-\mathbf{r}_{2}$, and $\mathbf{r}_{12}=\mathbf{r}_{2}-\mathbf{r}_{1}$. The same diagram applies for the forces between two point dipoles if spheres $\mathbf{M}_{1}$ and $\mathbf{M}_{2}$ are replaced by dipoles $\mathbf{m}_{1}$ and $\mathbf{m}_{2}$ at the same locations.

Equations (15) and (17) and a little algebra reveal that the net torque on an isolated pair of dipoles vanishes identically

$$
\tau_{12}^{A}+\tau_{21}^{A}+\tau_{12}^{B}+\tau_{21}^{B}=0 .
$$

The torque supplied by $\tau_{12}^{A}$ and $\tau_{21}^{A}$ therefore cancels the torque supplied by $\tau_{12}^{B}$ and $\tau_{21}^{B}$, and the angular momentum is conserved. Thus, an isolated dipole-dipole system does not spontaneously rotate, and there is no exchange between mechanical and electromagnetic momentum. Such exchanges have been the subject of considerable study. ${ }^{29,34-36}$

\section{FORCE BETWEEN SPHERES}

We now come to the crux of this article, the force between two uniformly magnetized spheres. We present four separate arguments that show that the force between uniformly magnetized spheres is identical to the force between point dipoles. While each proof is sufficient to show this equivalence, each utilizes different concepts from mechanics and electromagnetism, and each has pedagogical value.

As seen in Fig. 1, we take sphere $i$ to have position $\mathbf{r}_{i}$, radius $a_{i}$, magnetization $\mathbf{M}_{i}$, and total dipole moment

$$
\mathbf{m}_{i}=\frac{4}{3} \pi a_{i}^{3} \mathbf{M}_{i}
$$

Its magnetic field is given by $\mathbf{B}_{i}=2 \mu_{0} \mathbf{M}_{i} / 3$ inside the sphere (for $\left|\mathbf{r}-\mathbf{r}_{i}\right|<a_{i}$ ) and by Eq. (5) outside the sphere. ${ }^{8,9}$ We treat $\mathbf{M}_{i}$ as spatially uniform and constant in time, neglecting any demagnetization by external fields. This assumption is appropriate for high coercivity materials. ${ }^{37,38}$

\section{A. Newton's third law}

A five-step argument involving Newton's third law shows that the force between two spheres with uniform magnetizations $\mathbf{M}_{1}$ and $\mathbf{M}_{2}$ is identical to the force between two point dipoles with corresponding magnetic moments $\mathbf{m}_{1}$ and $\mathbf{m}_{2}$, located at the same positions and with the same magnetic orientations as the spheres. Figure 2 illustrates the argument. In Fig. 2(a), as in the preceding discussion, $\mathbf{F}_{12}$ represents the force of dipole 1 on dipole 2 . This force is produced by the field $\mathbf{B}_{1}$ of dipole 1. In Fig. 2(b), sphere 1 produces the same field $\mathbf{B}_{1}$, and therefore exerts the same force $\mathbf{F}_{12}$ on dipole 2. Newton's third law is shown in Fig. 2(c), giving the 
(a) $\mathbf{m}_{1}$
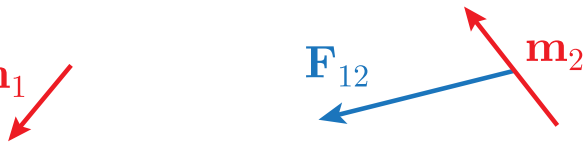

(b)
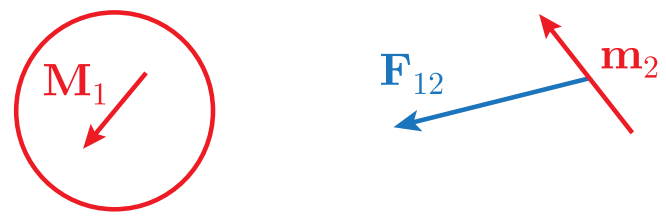

(c)

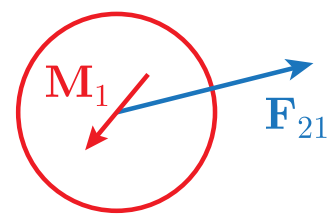

(d)

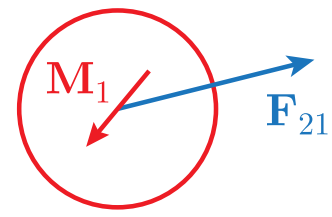

(e)

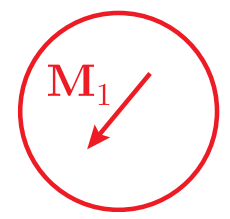

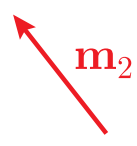
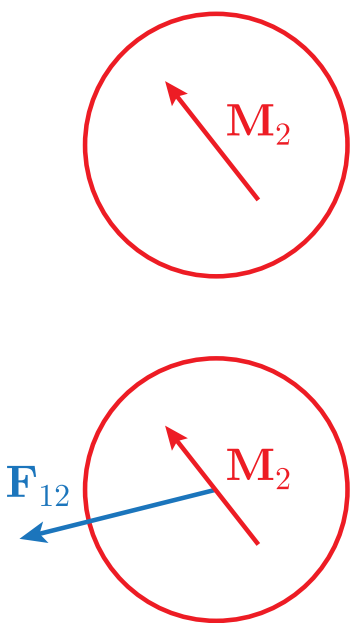

Fig. 2. Diagram illustrating the five steps of the Newton's third law argument showing that the force between two uniformly magnetized spheres is identical to the force between two point dipoles.

force $\mathbf{F}_{21}=-\mathbf{F}_{12}$ of dipole 2 on sphere 1. This force is produced by the field $\mathbf{B}_{2}$ of dipole 2. Sphere 2, as shown in Fig. 2(d), produces the same field $\mathbf{B}_{2}$ as dipole 2 (outside of sphere 2), and therefore exerts the same force $\mathbf{F}_{21}$ on sphere 1. Lastly, to complete the argument, we again apply Newton's third law to show that the force $\mathbf{F}_{12}=-\mathbf{F}_{21}$, shown in Fig. 2(e), of sphere 1 on sphere 2 is identical to the force of dipole 1 on dipole 2 [Fig. 2(a)].

\section{B. Direct integration}

The force between two uniformly magnetized spheres can be determined by first integrating the energy density $-\mathbf{M}_{j} \cdot \mathbf{B}_{i}$ associated with sphere $j$ sitting in the magnetic field $\mathbf{B}_{i}$, giving the total interaction energy

$$
U_{i j}=-\int_{j} \mathbf{M}_{j} \cdot \mathbf{B}_{i} d V=-\mathbf{M}_{j} \cdot \int_{j} \mathbf{B}_{i} d V .
$$

Here, the integral is over the volume of sphere $j$ and the second equality exploits the uniformity of $\mathbf{M}_{j}$. If all sources of a magnetic field lie outside a particular sphere, then the spatial average of the field over the sphere is given by the value of the field at the sphere center, ${ }^{39}$ thus

$$
\int_{j} \mathbf{B}_{i} d V=\frac{4}{3} \pi a_{j}^{3} \mathbf{B}_{i}\left(\mathbf{r}_{j}\right) .
$$

Equations (19)-(21) give $U_{i j}=-\mathbf{m}_{j} \cdot \mathbf{B}_{i}\left(\mathbf{r}_{j}\right)$, which replicates Eq. (7). Thus, the energy of interaction between the two spheres is identical to the energy of interaction between two point dipoles. The associated force $\mathbf{F}_{i j}=-\nabla_{j} U_{i j}$ of sphere $i$ on sphere $j$ is therefore identical to the force between two point dipoles, and obeys Newton's third law.

\section{Field energy}

We can also show the force equivalence by integrating the magnetic energy density $B^{2} / 2 \mu_{0}$ over all space, giving the total energy

$$
\begin{aligned}
U\left(\mathbf{r}_{1}, \mathbf{r}_{2}\right) & =\frac{1}{2 \mu_{0}} \int\left(\mathbf{B}_{1}+\mathbf{B}_{2}\right)^{2} d V \\
& =\frac{1}{2 \mu_{0}} \int\left(B_{1}^{2}+2 \mathbf{B}_{1} \cdot \mathbf{B}_{2}+B_{2}^{2}\right) d V .
\end{aligned}
$$

Because the magnetic energy of a single dipole does not depend on its location in space, the self-energy integrals $\left(2 \mu_{0}\right)^{-1} \int B_{i}^{2} d V$ do not depend on $\mathbf{r}_{i}$. Therefore, the force $\mathbf{F}_{i j}=-\nabla_{j} U$ on sphere $j$ depends only on the interaction energy

$$
\begin{aligned}
U_{\text {int }} & =\frac{1}{\mu_{0}} \int \mathbf{B}_{1} \cdot \mathbf{B}_{2} d V \\
& =\frac{1}{\mu_{0}}\left(\int_{1}+\int_{2}+\int_{\text {outside }}\right) \nabla \varphi_{1} \cdot \nabla \varphi_{2} d V,
\end{aligned}
$$

where we have inserted Eq. (3), and we have separated the integral over all space into integrals over sphere 1, sphere 2, and the region outside of both spheres.

For the integral over sphere 1 , we use $\nabla^{2} \varphi_{2}=0$ and the divergence theorem to write

$$
\begin{aligned}
\int_{1} \nabla \varphi_{1} \cdot \nabla \varphi_{2} d V & =\int_{1} \nabla \cdot\left(\varphi_{1} \nabla \varphi_{2}\right) d V \\
& =\int_{S_{1}} \varphi_{1} \nabla \varphi_{2} \cdot \hat{\mathbf{n}}_{1} d A
\end{aligned}
$$

where $S_{1}$ denotes the integral over the surface of sphere 1 , and $\hat{\mathbf{n}}_{1}$ is the unit vector directed normally outward from the sphere's surface. The quantity $\varphi_{1} \nabla \varphi_{2}$ is continuous across this surface, and the surface values of $\varphi_{1}$ and $\varphi_{2}$ are identical to the potentials of point dipoles. Therefore, the integral over sphere 1 is the same as it would be if the spheres were replaced by equivalent point dipoles. Similarly, the integral over sphere 2 is the same as for equivalent point dipoles. Because the fields outside of both spheres match the fields of 
equivalent point dipoles, all three integrals in Eq. (23) are the same for point dipoles as for uniformly magnetized spheres. Therefore, the force $\mathbf{F}_{i j}=-\nabla_{j} U_{\text {int }}$ must also be the same.

\section{Stress tensor}

The total force on an object can be calculated by integrating the Maxwell stress tensor over an arbitrary surface surrounding the object. ${ }^{40,41}$ The magnetic stress tensor depends only on the magnetic field. The field outside a uniformly magnetized sphere is the same as the field of an equivalent point dipole located at its center. The total field produced by and outside of two uniformly magnetized spheres, and the associated stress tensor, is the same as that of two point dipoles. Therefore, the force between spheres must be the same as the force between point dipoles.

\section{TORQUE BETWEEN SPHERES}

Here, we calculate the torque $\tau_{i j}$ of sphere $i$ on sphere $j$. This torque has two contributions, as before. The first arises from sphere $j$ residing in the field of sphere $i$ and can be obtained by integrating the torque density $\mathbf{M}_{j} \times \mathbf{B}_{i}$ over the volume of sphere $j$, giving

$$
\tau_{i j}^{A}=\int_{j} \mathbf{M}_{j} \times \mathbf{B}_{i} d V=\mathbf{M}_{j} \times \int_{j} \mathbf{B}_{i} d V .
$$

Invoking Eqs. (19) and (21) gives $\tau_{i j}^{A}=\mathbf{m}_{j} \times \mathbf{B}_{i}\left(\mathbf{r}_{j}\right)$, which replicates Eq. (14). Thus, $\tau_{i j}^{A}$ between two uniformly magnetized spheres is identical to the torque between two point magnetic dipoles.

The second contribution arises from the force of sphere $i$ on sphere $j$, which can be obtained from the interaction energy density $u_{i j}=-\mathbf{M}_{j} \cdot \mathbf{B}_{i}$ according to

$$
\mathbf{F}_{i j}=-\int_{j} \nabla u_{i j} d V
$$

This gives the same force as $\mathbf{F}_{i j}=-\nabla_{j} U_{i j}$, with $U_{i j}$ given by Eq. (7). The torque follows by integrating the torque density $\mathbf{r} \times\left(-\nabla u_{i j}\right)$ over the volume of sphere $j$ to give

$$
\tau_{i j}^{B}=-\int_{j} \mathbf{r} \times \nabla u_{i j}(\mathbf{r}) d V
$$

Rewriting this expression using the position $\mathbf{r}^{\prime}=\mathbf{r}-\mathbf{r}_{j}$ relative to the center of sphere $j$ gives

$$
\begin{aligned}
\tau_{i j}^{B} & =-\int_{j}\left(\mathbf{r}^{\prime}+\mathbf{r}_{j}\right) \times \nabla u_{i j}(\mathbf{r}) d V \\
& =-\int_{j} \mathbf{r}^{\prime} \times \nabla^{\prime} \Psi\left(\mathbf{r}^{\prime}\right) d V^{\prime}-\mathbf{r}_{j} \times \int_{j} \nabla u_{i j}(\mathbf{r}) d V
\end{aligned}
$$

where $\Psi\left(\mathbf{r}^{\prime}\right)=u_{i j}\left(\mathbf{r}^{\prime}+\mathbf{r}_{j}\right)$. Converting the first integral into a surface integral gives

$$
\begin{aligned}
\int_{j} \mathbf{r}^{\prime} \times \nabla^{\prime} \Psi d V^{\prime} & =-\int_{j} \nabla^{\prime} \times\left(\Psi \mathbf{r}^{\prime}\right) d V^{\prime} \\
& =-\int_{S_{j}} \hat{\mathbf{n}}_{j} \times \mathbf{r}^{\prime} \Psi d A^{\prime}
\end{aligned}
$$

Since $\mathbf{r}^{\prime}$ is parallel to the unit normal vector $\hat{\mathbf{n}}_{j}$, their cross product is zero and the integral vanishes. Combining Eqs. (26) and (28) then gives

$$
\tau_{i j}^{B}=\mathbf{r}_{j} \times \mathbf{F}_{i j}
$$

which is identical to Eq. (16). Thus, the torque associated with the force on a uniformly magnetized sphere in a dipole field is the same as the torque on the corresponding point dipole. These results ensure that the net torque on an isolated two-sphere system is zero, as seen earlier for point dipoles.

\section{SUMMARY}

We have demonstrated that the energy, forces, and torques between two uniformly magnetized spheres are identical to those between two point magnetic dipoles. This equivalence immediately extends to uniformly polarized spheres and point electric dipoles because the fields, forces, and torques of electric dipoles have the same mathematical forms as their magnetic counterparts, and the field outside of a uniformly polarized sphere is identical to the electric dipole field.

\section{ACKNOWLEDGMENTS}

The authors gratefully acknowledge support from NSF Grant No. 1332265, discussions with W. Farrell Edwards on the applicability of Newton's third law to magnetostatic interactions, correspondence with David Vokoun about calculations of the force between magnet spheres in special cases, correspondence with Marco Beleggia about the potential theory argument, and discussions with Kirk McDonald and David Griffiths regarding the point-sphere equivalence. The authors are especially grateful to David Griffiths for suggesting the stress tensor argument to us, and to Kirk McDonald for pointing out a mean-value argument ${ }^{39}$ that simplifies some of the calculations.

${ }^{1}$ J. D. Jackson, Classical Electrodynamics, 2nd ed. (Wiley, New York, 1975), Sec. 5.6, pp. 180-184.

${ }^{2} \mathrm{G}$. Akoun and J. Yonnet, "3D analytical calculation of the forces exerted between two cuboidal magnets," IEEE Trans. Magn. 20, 1962-1964 (1984).

${ }^{3}$ A. Kruusing, "Optimizing magnetization orientation of permanent magnets for maximal gradient force," J. Magn. Magn. Mater. 234, 545-555 (2001).

${ }^{4}$ D. Vokoun, M. Beleggia, L. Heller, and P. Sittner, "Magnetostatic interactions and forces between cylindrical permanent magnets," J. Magn. Magn. Mater. 321, 3758-3763 (2009).

${ }^{5}$ J. S. Agashe and D. P. Arnold, "A study of scaling and geometry effects on the forces between cuboidal and cylindrical magnets using analytical force solutions," J. Phys. D: Appl. Phys. 41, 105001 (2008); J. S. Agashe and D. P. Arnold, "Corrigendum: A study of scaling and geometry effects on the forces between cuboidal and cylindrical magnets using analytical force solutions," J. Phys. D: Appl. Phys. 42, 099801 (2009).

${ }^{6} \mathrm{~S}$. Sanz, L. Garcia-Tabares, I. Moya, D. Obradors, and F. Toral, "Evaluation of magnetic forces in permanent magnets," IEEE Trans. Appl. Supercond. 20, 846-850 (2010).

${ }^{7}$ M. Beleggia, S. Tandon, Y. Zhi, and M. De Graef, "On the magnetostatic interactions between nanoparticles of arbitrary shape," J. Magn. Magn. Mater. 278, 270-284 (2004).

${ }^{8}$ R. K. Wangsness, Electromagnetic Fields, 2nd ed. (Wiley, New York, 1986), p. 326.

9J. D. Jackson, Classical Electrodynamics, 2nd ed. (Wiley, New York, 1975), p. 195.

${ }^{10}$ M. Varón, M. Beleggia, T. Kasama, R. J. Harrison, R. E. DuninBorkowski, V. F. Puntes, and C. Frandsen, "Dipolar magnetism in ordered 
and disordered low-dimensional nanoparticle assemblies," Sci. Rep. 3, 1-5 (2013).

${ }^{11}$ G. Helgesen, T. T. Skjeltorp, P. M. Mors, R. Botet, and R. Jullien, "Aggregation of magnetic microspheres: Experiments and simulations," Phys. Rev. Lett. 61(15), 1736-1739 (1988).

${ }^{12}$ D. A. Richter, "Expert report, Teaching geometry with magnet sphere kits, in the matter of Zen Magnets, LLC, CPSC Docket No. 12-2," Item 124, Exhibit 3, 2014 <http://www.cpsc.gov/en/Recalls/Recall-Lawsuits/ Adjudicative-Proceedings/> (accessed 9 February, 2016).

${ }^{13}$ B. F. Edwards, "Expert report: Educational value of neodymium magnet spheres in the matter of Zen Magnets, LLC, CPSC Docket No. 12-2," Item 124, Exhibit 4, 2014, <http://www.cpsc.gov/en/Recalls/Recall-Lawsuits/ Adjudicative-Proceedings/> (accessed February 9, 2016).

${ }^{14}$ The Zen Gallery, curated by Shihan Qu, shows photos of various magnetic sculptures including models of fractals, molecules, lattices, and Platonic solids $<$ http://zenmag nets.com/gallery/ $>$ (accessed February 9, 2016).

${ }^{15}$ Typing "Zen Magnets" into the YouTube search field at $<$ https:// www.youtube.com> identifies over 90,000 videos describing various magnet structures (accessed March 23, 2016). As of August 22, 2014, the most popular of these had a total view count exceeding $145 \times 10^{6}$ (Ref. 13, Appendix D).

${ }^{16}$ On November 22, 2016, the United States Court of Appeals for the Tenth Circuit ended a two-year sales ban by the United States Consumer Product Safety Commission on sets of small high-powered magnets, ruling that the factual findings on which the ban was based were incomplete and inadequately explained <https://drive.google.com/file/d/0Bw7DdocNZGQ gbWlON2loT2FfQzA/view $>$. Magnet sets marketed as desk toys may now be purchased in the United States from suppliers including $(<\mathrm{http}: / /$ www.zenmagnets.com $>, \quad<$ http://www.buckyballsstore.com $>, \quad$ and $<$ http://www.neoballs.com $>$, and may also be purchased from industrial suppliers including $\quad<$ http://www.kjmagnetics.com $>, \quad<$ http:// www.alibaba.com $>$, and <http://www.magnet4less.com $>$ (accessed December 29, 2016).

${ }^{17}$ B. F. Edwards and John M. Edwards, "Dynamical interactions between two uniformly magnetized spheres," Eur. J. Phys. 38, 015205 (2017).

${ }^{18}$ J. M. Edwards, MagPhyx Simulation and Visualization Software, $<$ http:// www2.cose.isu.edu/edwajohn/MagPhyx > (accessed March 11, 2016). This web-based software simulates the 2D motion of a uniformly magnetized sphere in response to the forces and torques supplied by a second uniformly magnetized sphere, held fixed. It is provided freely to the physics community for education and exploration.

${ }^{19} \mathrm{M}$. Beleggia and M. De Graef, "General magnetostatic shape-shape interactions," J. Magn. Magn. Mater. 285, L1-L10 (2005).

${ }^{20} \mathrm{D}$. Vokoun and M. Beleggia, "Forces between arrays of permanent magnets of basic geometric shapes," J. Magn. Magn. Mater. 350, 174-178 (2014). This publication quotes the result of an unpublished calculation of the magnetic interaction between two identical spheres with parallel magnetizations. Details of this calculation were sent privately to us by D. Vokoun on February 8, 2016.

${ }^{21} \mathrm{~W}$. Booth and B. Edwards (unpublished).

${ }^{22}$ D. Griffiths, Introduction to Electrodynamics, 3rd ed. (Pearson Education, Upper Saddle River, 1999), p. 282.
${ }^{23}$ K. W. Young, P. B. Landecker, and D. D. Villani, "An analytic solution for the force between two magnetic dipoles," Magn. Electr. Sep. 9, 39-52 (1998).

${ }^{24}$ T. H. Boyer, "The force on a magnetic dipole," Am. J. Phys. 56, 688-692 (1988).

${ }^{25}$ K. R. Brownstein, "Force exerted on a magnetic dipole," Am. J. Phys. 61, 940-941 (1993).

${ }^{26}$ J. B. Greene and F. G. Karioris, "Force on a magnetic dipole," Am. J. Phys. 39, 172-175 (1971).

${ }^{27}$ L. Vaidman, "Torque and force on a magnetic dipole," Am. J. Phys. 58, 978-983 (1990).

${ }^{28}$ R. P. Feynman, R. B. Leighton, and M. Sands, The Feynman Lectures on Physics (Addison-Wesley, Boston, MA, 1964), Vol. II, pp. 26-25. Here, Feynman discusses the example of the non-central force between two positively charged particles, one moving in the $+x$ direction and the other moving in the $+y$ direction. At the instant that particle 1 is at the coordinate origin and particle 2 is on the $+y$ axis, the electric forces are central and repulsive, but particle 1 exerts a non-central magnetic force on particle 2 in the $+x$ direction, while particle 2 exerts no magnetic force on particle 1. Thus, Newton's third law does not apply, raising a paradox about the origin of the extra mechanical momentum.

${ }^{29}$ A. Caprez and H. Batelaan, "Feynman's relativistic electrodynamics paradox and the Aharonov-Bohm effect," Found. Phys. 39, 295-306 (2009). Here, the authors show that the extra mechanical momentum of Feynman's paradox is hidden in the electromagnetic fields.

${ }^{30}$ J. B. Marion and S. T. Thornton, Classical Dynamics of Particles and Systems, 4th ed. (Saunders College Publishing, Fort Worth, TX, 1995), p. 50.

${ }^{31}$ Reference 22, p. 246.

${ }^{32}$ D. J. Griffiths, “Dipoles at rest," Am. J. Phys. 60, 979-987 (1992).

${ }^{33} \mathrm{We}$ ignore the torque on a sphere due to the electric dipole moment that it acquires from its motion. Point magnetic dipoles in uniform motion through a uniform magnetic field acquire an electric dipole moment that interacts with the magnetic field to give an additional torque on the dipole. This torque is proportional to $v^{2} / c^{2}$, we ignore it for our non-relativistic investigations. See David J. Griffiths and V. Hnizdo, "The torque on a dipole in uniform motion," Am. J. Phys. 82, 251-254 (2014).

${ }^{34}$ D. J. Griffiths and V. Hnizdo, "Mansuripur's paradox," Am. J. Phys. 81, 570-574 (2013).

${ }^{35} \mathrm{~V}$. Hnizdo, "Hidden mechanical momentum and the field momentum in stationary electromagnetic and gravitational systems," Am. J. Phys. 65, 515-518 (1997)

${ }^{36} \mathrm{~T}$. H. Boyer, "Classical interaction of a magnet and a point charge: The Shockley-James paradox,” Phys. Rev. E 91, 013201 (2015).

${ }^{37}$ R. K. Wangsness, Electromagnetic Fields, 2nd ed. (Wiley, New York, 1986), p. 340.

${ }^{38}$ Coercivity, Wikipedia, <http://en.wikipedia.org/wiki/Coercivity $>$ (accessed February 16, 2016).

${ }^{39} \mathrm{~B}$. Y. Hu, "Averages of static electric and magnetic fields over a spherical region: A derivation based on the mean-value theorem," Am. J. Phys. 68, 1058-1060 (2000).

${ }^{40}$ J. Vanderlinde, Classical Electromagnetic Theory, 1st ed. (Wiley, New York, 1993), pp. 81-84.

${ }^{41}$ Reference 22, pp. 351-354. 